\title{
Avaliação da empatia de graduandos de enfermagem
}

Empathy assessment among nursing undergraduates Evaluación de la empatía de estudiantes universitarios de enfermería

\author{
Isabel Amélia Costa Mendes ${ }^{1}$ (i) https://orcid.org//0000-0002-0704-4319 \\ Suzel Regina Ribeiro Chavaglia² ${ }^{2}$ in htps:///orcid.org/0000-0001-7033-0185 \\ Simone de Godoy ${ }^{1}$ (I) https://orcid.org/0000-0003-0020-7645 \\ Ítalo Rodolfo Silva ${ }^{3}$ io nttps://orcid.org/0000-0002-2882-1877 \\ Emerson Willian Santos Almeida ${ }^{1}$ id https://orcid.orgy/0000-0002-6846-021X \\ Mirella Castelhano Souza ${ }^{4}$ io https://orcid.org/0000-0001-9036-3304
}

Como citar:

Mendes IA, Chavaglia SR, Godoy S, Silva IR, Almeida EW, Souza MC. Avaliação da empatia de graduandos de enfermagem. Acta Paul Enferm. 2021;34:eAPE002235.

DOI

http://dx.doi.org/10.37689/actaape/2021A0002235

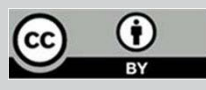

Descritores

Empatia; Ensino de enfermagem; Comunicação; Relações interpessoais

Keywords

Empathy; Nursing education; Communication; Interpersonal relations

Descriptores

Empatía; Educación en enfermeiría Comunicación Relaciones interpersonales

\section{Submetido \\ 13 de Agosto de 2020 \\ Aceito \\ 3 de Dezembro de 2020}

Autor correspondente

Isabel Amélia Costa Mendes

E-mail: iamendes@usp.br

\section{Resumo}

Objetivo: Verificar o grau de empatia de estudantes de enfermagem de um curso de universidade pública de um estado da região Sudeste do Brasil e avaliar seu perfil em relação aos domínios: Tomada de Perspectiva, Flexibilidade Interpessoal, Altruísmo e Sensibilidade Afetiva.

Métodos: Estudo exploratório, desenvolvido com estudantes de graduação em enfermagem, utilizando-se o Inventário de Empatia (E), instrumento composto de 40 questões empregadas para avaliar quatro dimensões da empatia.

Resultados: Do total de 193 participantes, 88,6 era do sexo feminino, sendo 78,7\% menores de 25 anos de idade e média de idade de 23,4 anos ( $\mathrm{DP}= \pm 4,8$ ). Quanto ao período do curso $89(46,1 \%)$ estavam entre o primeiro e quarto, $74(38,4 \%)$ entre o quinto e oitavo, e $30(15,5 \%)$ eram do nono e décimo semestre. Verificou-se que os graduandos possuem habilidade de empatia.

Conclusão: Os graduandos de enfermagem apresentaram escores acima da média em todas as dimensões avaliadas pelo IE, demonstrando que possuem um alto índice de empatia.

\section{Abstract}

Objective: To verify the degree of empathy of students attending the nursing program of a public university located in southeastern Brazil and assess their profile regarding the domains: Perspective Taking, Interpersonal Flexibility, Altruism, and Affective Sensibility.

Methods: Exploratory study conducted among undergraduate nursing students, using the Empathy Inventory (E)), a 40-item instrument designed to assess four dimensions of empathy.

Results: Of the total of 193 participants, $88.6 \%$ were women, $78.7 \%$ were under 25 years of age, with a mean of 23.4 years old $(\mathrm{SD}= \pm 4.8$ ). Regarding the semester, 89 (46.\%) were attending between the first and fourth semesters, 74 (38.4\%) between the fifth and eighth semesters, and 30 (15.5\%) were attending the ninth or tenth semester. The undergraduate students presented empathy skills.

Conclusion: The nursing undergraduate students scored above the mean in all the dimensions assessed by the El, showing a high level of empathy.

\section{Resumen}

Objetivo: Verificar el nivel de empatía de estudiantes de enfermería de una carrera de universidad pública en un estado de la región Sudeste de Brasil y evaluar su perfil con relación a los dominios: Toma de perspectiva, Flexibilidad interpersonal, Altruismo y Sensibilidad afectiva. 
Métodos: Estudio exploratorio, realizado con estudiantes universitarios de enfermería, utilizando el Inventario de Empatía (IE), instrumento compuesto por 40 preguntas para evaluar cuatro dimensiones de la empatía.

Resultados: Del total de 193 participantes, 88,6 eran de sexo femenino, el 78,7 \% menores de 25 años y un promedio de edad de 23,4 años (DP $= \pm 4,8$ ). Con relación al período de la carrera, 89 estudiantes (46,1\%) estaban entre el primer y cuarto semestre, 74 (38,4 \%) entre el quinto y el octavo y 30 (15,5 \%) cursaban el noveno y décimo semestre. Se verificó que los estudiantes tienen habilidades de empatía.

Conclusión: Los estudiantes universitarios de enfermería presentaron puntuaciones superiores al promedio en todas las dimensiones evaluadas por el IE, lo que demuestra que poseen un alto índice de empatía.

\section{Introdução}

Amplamente aceita como essencial para a relação de ajuda, empatia pode ser definida, mas não pode ser descrita; é somente vivenciada e reconhecida. ${ }^{(1)}$ Pode ser vista como intrínseca às profissóes da área da saúde, uma vez que enfermeiros, médicos, fisioterapeutas, dentre outros, devem utilizá-la posicionando-se no lugar dos pacientes para fundamentar suas ações profissionais. ${ }^{(2)}$

Acredita-se que a empatia corresponde à capacidade de aprimoramento da compreensão, expressando este entendimento de tal maneira que a outra pessoa se sinta compreendida, o que transforma a empatia numa habilidade social. ${ }^{(3,4)}$ A frequência de estudos sobre o tema tem se intensificado nos anos recentes, em várias carreiras da área da saúde, como Medicina, ${ }^{(5)}$ Educação, ${ }^{(6)}$ variadas profissões de saúde, ${ }^{(7)}$ orientação cultural, ${ }^{(8,9)}$ marketing, ${ }^{(10)}$ Enfermagem, ${ }^{(11-15)}$ laboratório de ética nos cuidados; ${ }^{(16)}$ Nutrição e dietética, ${ }^{(17)}$ dentre vários.

No âmbito da Enfermagem as experiências que conduzem ao desenvolvimento de situaçóes empáticas têm início na formação do estudante de graduação, assim como nas práticas simuladas e permeiam toda a trajetória do profissional de enfermagem. É um componente crítico da relação enfermeiro-paciente, que exige autoconhecimento do profissional para que possa compreender os sentimentos do paciente. ${ }^{(18)}$

Em nosso meio o perfil empático de profissionais de enfermagem ${ }^{(12)}$ foi avaliado utilizando o Inventário de Empatia. ${ }^{(3)}$ Para o presente estudo foi adotada a aplicação desse mesmo instrumento, com os objetivos de verificar o grau de empatia de estudantes de enfermagem de um curso de universidade pública de um estado da regiáo Sudeste do Brasil e avaliar seu perfil em relaçáo aos domínios: Tomada de Perspectiva, Flexibilidade Interpessoal, Altruísmo e Sensibilidade Afetiva.

\section{Métodos}

Estudo exploratório realizado com estudantes de graduação em enfermagem. Para a coleta de dados foram respeitados os preceitos éticos e legais da pesquisa com seres humanos. Nesse contexto, o estudo teve início somente após a autorização livre e esclarecida dos participantes, a partir da assinatura do Termo de Consentimento Livre e Esclarecido (Protocolo 1348/2011). Ademais, a pesquisa foi realizada mediante autorização do docente responsável pela disciplina/aula na ocasião da coleta de dados.

Foram critérios de inclusão: ser estudante do curso de graduação em enfermagem, a partir do primeiro período/semestre do tempo de integralização do referido curso. Já, como critérios de exclusão, os estudantes de licença médica ou ausentes na data da coleta de dados. $\mathrm{O}$ processo de recrutamento se deu por conveniência, cujo convite para a pesquisa ocorreu momentos antes da coleta.

Os dados foram coletados no mês de março de 2019 com o apoio de dois questionários, sendo um para a caracterização da amostra (sexo, idade e período do curso) e o Inventário de Empatia (IE), ${ }^{(3)}$ cujo consentimento para uso foi obtido pela pesquisadora responsável.

O IE contempla 40 questóes que avaliam quatro dimensóes da empatia: Tomada de Perspectiva, Flexibilidade Interpessoal, Altruísmo e Sensibilidade Afetiva. ${ }^{(19)}$ As respostas são indicadas em uma escala tipo Likert de 5 pontos, onde (1) corresponde a nunca, (2) raramente, (3) regularmente, (4) quase sempre e (5) sempre.

Dos 40 itens, 17 são reversos, portanto, as respostas desses itens foram invertidas para a obtenção do escore final. Assim, quando a resposta dada foi 5 , substitui-se por 1 ou vice-versa; 4 por 2 ou vice-versa e a resposta 3 foi mantida. 
Os dados coletados foram codificados e duplamente digitados em planilhas do aplicativo Excel e, após a verificação de consistência, foram exportados e analisados no programa IBM SPSS Statistics versão 25.

$\mathrm{Na}$ análise estatística descritiva, foram apresentadas as frequências absoluta e relativa das variáveis sexo, curso, semestre que o aluno está cursando e os valores mínimo, máximo, mediana, média e desvio padrão para as variáveis idade e para cada componente do IE. Para o IE foram somados os totais dos escores de seus componentes e verificada a consistência interna com o cálculo dos valores do Alfa de Cronbach total e para cada componente.

Para verificar a relaçáo entre as variáveis idade e ano do curso com os componentes da escala aplicou-se o Coeficiente de Correlação de Spearman e para comparar os escores dos quatro componentes entre os sexos aplicou-se o teste Mann-Whitney, uma vez que as variáveis submetidas ao teste de Shapiro-Wilk não apresentaram normalidade.

Para a análise estatística adotou-se o nível de significância de $\mathrm{p}=0,05$.

\section{Resultados}

Participaram do estudo 193 graduandos de enfermagem de uma universidade pública de município do interior do sudeste brasileiro. A maioria dos participantes era do sexo feminino $171(88,6 \%)$ possuía menos de 25 anos $152(78,7 \%)$ e média de idade de 23,4 anos (DP $= \pm 4,8)$. Quanto ao período do curso $89(46,1 \%)$ estavam entre o primeiro e quarto, $74(38,4 \%)$ entre o quinto e oitavo, e 30 $(15,5 \%)$ eram do nono e décimo semestre.

Os coeficientes Alfa de Cronbach para cada um dos componentes e para a escala total do IE mostraram valores satisfatórios (Tabela 1), semelhantes aos obtidos no estudo original que, com base nos critérios estabelecidos, considera satisfatório valor maior ou igual a 0,70 .

Com a aplicação do teste de Mann-Whitney, os valores médios obtidos para cada um dos componentes do Inventário de Empatia foram comparados entre os sexos, mostrando diferença significante entre sexo feminino e Sensibilidade Afetiva $(\mathrm{p}=0,010)$ (Tabela 2).
Tabela 1. Escores do Inventário de Empatia atribuídos pelos graduandos de enfermagem $(n=193)$ de uma instituição pública

\begin{tabular}{|c|c|c|c|c|c|}
\hline Fatores & Mínimo & Máximo & Mediana & Média(DP) & $\begin{array}{c}\text { Alfa de } \\
\text { Cronbach }\end{array}$ \\
\hline Tomada de Perspectiva & 1 & 5 & 4 & $3,8(1,0)$ & 0,82 \\
\hline Flexibilidade Interpessoal & 1 & 5 & 3 & $3,2(1,1)$ & 0,76 \\
\hline Altruísmo & 1 & 5 & 4 & $3,7(1,1)$ & 0,70 \\
\hline Sensibilidade Afetiva & 1 & 5 & 4 & $4,2(1,0)$ & 0,72 \\
\hline Total & 1 & 5 & 4 & $3,7(1,1)$ & 0,81 \\
\hline
\end{tabular}

Tabela 2. Comparação dos escores médios dos componentes do Inventário de Empatia, entre os graduandos de enfermagem do sexo feminino $(n=171)$ e masculino $(n=22)$ de uma instituição pública

\begin{tabular}{|c|c|c|c|c|c|}
\hline Dimensões/Sexo & Mínimo & Máximo & Mediana & Média(DP) & $p$-value* \\
\hline Tomada de Perspectiva & & & & & 0,497 \\
\hline Feminino & 2,6 & 5,0 & 3,8 & $3,8(0,5)$ & \\
\hline Masculino & 1,9 & 4,9 & 3,9 & $3,6(0,8)$ & \\
\hline Flexibilidade Interpessoal & & & & & 0,633 \\
\hline Feminino & 1,2 & 4,6 & 3,2 & $3,2(0,6)$ & \\
\hline Masculino & 2,2 & 4,6 & 3,3 & $3,3(0,6)$ & \\
\hline Altruísmo & & & & & 0,984 \\
\hline Feminino & 1,8 & 4,8 & 3,8 & $3,7(0,6)$ & \\
\hline Masculino & 3,0 & 4,7 & 3,9 & $3,7(0,6)$ & \\
\hline Sensibilidade Afetiva & & & & & 0,010 \\
\hline Feminino & 2,6 & 5,0 & 4,3 & $4,2(0,5)$ & \\
\hline Masculino & 3,1 & 4,9 & 3,8 & $3,9(0,5)$ & \\
\hline Total & & & & & 0,380 \\
\hline Feminino & 2,8 & 4,7 & 3,8 & $3,8(0,3)$ & \\
\hline Masculino & 2,8 & 4,6 & 3,8 & $3,7(0,5)$ & \\
\hline
\end{tabular}

O componente Sensibilidade Afetiva apresentou correlação inversa com a variável período do curso $(p=0,032)$, ou seja, quanto mais avançado o graduando está no curso, menor é a sua pontuação (Tabela 3). As demais dimensóes não mostraram correlação com as variáveis idade e período do curso.

Tabela 3. Coeficiente de Correlação de Spearman entre os escores médios das dimensões do Inventário de Empatia e a idade e período que está cursando, dos graduandos de enfermagem $(n=193)$ de uma instituição pública

\begin{tabular}{lcccc}
\hline \multirow{2}{*}{ Dimensões } & \multicolumn{2}{c}{ Idade } & \multicolumn{2}{c}{ Período do Curso } \\
& Coeficiente & $\boldsymbol{p}$-value & Coeficiente & $\boldsymbol{p}$-value \\
\hline Tomada de Perspectiva & $-0,087$ & 0,230 & $-0,141$ & 0,050 \\
Flexibilidade Interpessoal & $-0,024$ & 0,739 & 0,065 & 0,371 \\
Altruísmo & $-0,119$ & 0,098 & $-0,094$ & 0,195 \\
Sensibilidade Afetiva & $-0,066$ & 0,365 & $-0,155$ & 0,032 \\
Total & $-0,121$ & 0,095 & $-0,118$ & 0,101 \\
\hline
\end{tabular}

\section{Discussão}

Os dados revelam que a pesquisa converge com o que é comumente descrito em investigaçóes com 
graduandos de enfermagem, em que há predomínio de participantes do sexo feminino e média de idade de 23 anos. ${ }^{(13,15)}$

Pode-se identificar ainda que os graduandos possuem uma boa habilidade de empatia, sendo essa considerada essencial para o desempenho profissional do enfermeiro e de outros profissionais da saúde. ${ }^{(2,5,7,10,13-17-21)}$

Em todas as dimensóes do IE, independente do sexo, os graduandos apresentaram escores acima da média. Cabe destacar que a dimensão referente a "Sensibilidade Afetiva" concentrou médias maiores de pontuação, apresentando diferença significante entre o sexo feminino e masculino $(\mathrm{p}=0,010)$, mostrando que as mulheres parecem ser mais atenciosas e cuidadosas com o estado emocional do outro, além de reconhecerem o que o outro precisa para se sentir melhor, exemplificado pelo maior percentual $(90,2 \%)$ de respostas ao item 34 "Durante uma conversação procuro demonstrar interesse pela outra pessoa, adotando uma postura atenta". Este resultado indicativo de maior sensibilidade afetiva não é incomum, tanto que enseja estudos questionando se a profissão de enfermagem seria atrativa para homens, ${ }^{(22)}$ já que se trata de uma carreira fortemente vinculada ao cuidar, o que pressupóe que enfermeiros sejam capazes de sentir empatia pela pessoa de quem cuida.

$\mathrm{Na}$ sequência, a dimensão "Tomada de Perspectiva" mostra que grande parte dos graduandos já adota postura cuidadosa e não têm dificuldades em compreender sentimentos e perspectivas diferentes das suas, sendo o item 33 "Costumo me colocar no lugar de uma pessoa que está me revelando um problema para ver como me sentiria e o que pensaria se a situação fosse comigo" aquele que detém a maior concentração de respostas $(81,8 \%)$.

Em relação ao "Altruísmo" os participantes demonstraram pouca tendência ao egoísmo, de maneira que o item 20 "Se alguém me deve algo, cobro-lhe a dívida imediatamente, mesmo que ele possa ter motivos que justifiquem o não pagamento" concentrou nas respostas nunca/raramente o posicionamento da maior parte $(84,9 \%)$ dos graduandos.

$\mathrm{Na}$ dimensão "Flexibilidade Interpessoal" os graduandos demonstraram que sabem aceitar pontos de vistas diferentes e buscam entendimento em situaçóes conflitantes, haja vista a maioria $(57,5 \%)$ de respostas em nunca/raramente no item 24 "Quando alguém age comigo de maneira hostil, respondo da mesma forma".

A consistência interna do IE foi verificada pelo alfa de Cronbach, resultando em 0,81 para a escala total; porém para as dimensóes Flexibilidade Interpessoal e Altruísmo os níveis não foram bons. Outros estudos que utilizaram o IE obtiveram boa consistência interna para todas as dimensóes. ${ }^{(4,23)}$

Sendo a maior parte dos graduandos do sexo feminino, identificou-se boas habilidades empáticas, conforme já identificado em outros estudos que avaliaram empatia entre universitários. ${ }^{(5-7,12-}$ 17,21) Tais resultados estão relacionados ao fato de que desde a infância as meninas desenvolvem os comportamentos de cuidar e confortar, sendo esses comportamentos também relacionados a prática de cuidados na enfermagem. Uma vez que a empatia é uma capacidade passível de ser desenvolvida e está relacionada à formação de vínculo, sensibilização e envolvimento entre duas ou mais pessoas, as meninas a desenvolvem desde a infância sob influência das práticas parentais de cuidado. ${ }^{(23)}$

É de se enfatizar que à medida que os cuidados de saúde aproximam-se cada vez mais de tecnologias, distanciando-se consequentemente das interaçôes com os pacientes, é vital que se atente para a manutenção da empatia no centro da interação com o paciente. É recomendável que os docentes de enfermagem desenvolvam abordagens criativas em termos de empatia também entre os estudantes, de modo que simulaçóes e cenários possam fomentar comportamento empático. ${ }^{(24)}$ De tal modo, mesmo em ambientes de ensino-aprendizagem controlados e fora dos cenários clínicos os docentes devem estar atentos para estimular o pensamento empático entre os estudantes e a prática da atitude empática em simulaçôes: em assim agindo o docente, que também atua de forma empática com os alunos, dá o exemplo e favorece a adoçáo e a incorporação do conceito da empatia pelos estudantes.

A identificação de estudantes de enfermagem com baixo nível de empatia já é indicador de cliente prioritário para treinamento e desenvolvimento 
mais aprofundado visando ajustes, tendo em vista o relacionamento interpessoal que ele terá com o paciente: esforços devem ser envidados por docentes para direcionamento da educação em enfermagem baseada em empatia , especialmente considerando-se que esta profissão abrange o relacionamento terapêutico. $^{(11)}$

Como limitação deste estudo é possível indicar que a porcentagem de graduandos dos últimos períodos do curso é pequena e não permite avaliar mudanças na atitude empática, o que nos leva a concluir que outros estudos mais amplos se fazem necessários.

\section{Conclusão}

Os resultados permitiram identificar que os graduandos de enfermagem desta pesquisa apresentaram escores acima da média em todas as dimensóes avaliadas pelo IE, demonstrando que possuem um alto índice de empatia. Dessa forma pode-se perceber que os graduandos já possuem habilidades essenciais para a prática profissional da enfermagem, destacando-se que na dimensão Sensibilidade Afetiva as mulheres apresentaram médias maiores de pontuação.

\section{Colaborações}

Mendes IAC, Chavaglia SRR, Godoy S, Silva IR, Almeida EWS e Souza MC colaboraram com a concepção e desenho do estudo, análise e interpretação dos dados, redação do artigo, revisão crítica do conteúdo intelectual e aprovação final da versão a ser publicada.

\section{Referências}

1. Tschudin V. Counselling skills for nurses. London: Bailliére; 1987.Vol. 2.

2. Larson $\mathrm{EB}, \mathrm{Yao} X$. Clinical empathy as emotional labor in the patientphysician relationship. JAMA. 2005;293(9):1100-6.

3. Falcone EM, Ferreira MC, Luz RC, Fernandes CS, Faria CA. D'Augustin $\mathrm{JF}$, et al. Inventário de empatia (IE): desenvolvimento e validação de uma medida brasileira. Aval Psicol. 2008;3(7):321-34.
4. Decety J, Jackson PL. The functional architecture of human empathy. Behav Cogn Neurosci Rev. 2004;3(2):71-100.

5. Shapiro J, Youm J, Kheriaty A, Pham T, Chen Y, Clayma R. The human kindness curriculum: An innovative preclinical initiative to highlight kindness and empathy in medicine. Educ Health (Abingdon). 2019;32(2):53-61.

6. Posti-Ahokas $H$. Empathy-based stories capturing the voice of female secondary school students in Tanzania. Int J Qual Stud Educ. 2013;26(10):1277-92.

7. Williams B, Brown T, McKenna L, Palermo C, Morgan P, Nestel D, et al. Student empathy levels across 12 medical and health professions: an interventional study. J Compassionate Health Care. 2015;2:4.

8. Formiga N, Souza MA. Type of cultural orientation and empathy in brazilians: verification of a theoretical model. Est Inter Psicol. 2012;3(2):139-61.

9. Dias OV, Vieira MA, Dias JP, Ramos LH. As dimensões da satisfação dos usuários do Programa Saúde da Família: confiabilidade e empatia. Acta Paul Enferm. 2011;24(2):225-31.

10. Escalas JE, Stern BB. Sympathy and empathy: emotional responses to advertising dramas. J Consum Res. 2003;29(4):566-78.

11. Brunero $S$, Lamont $S$, Coates M. A review of empathy education in nursing. Nurs Inq. 2010;17(1):65-74.

12. Trevizan MA, Almeida RG, Souza MC, Mazzo A, Mendes IA, Martins JC. Empathy in Brazilian nursing professionals: a descriptive study. Nurs Ethics. 2015;22(3):367-76.

13. Mendes IA, Trevizan MA, Souza MC, Souza-Junior VD, de Godoy S, Ventura CA, et al. Empathic profile of nursing freshmen. Nurs Ethics. 2019;26(7-8):2298-305.

14. Souza MC, Godoy S, Santos SS, Trevizan MA, Silveira RC, Mendes IA. Como medir o grau empático de graduandos de enfermagem? Uma revisão integrativa. Texto Contexto Enferm. 2019;28:e20180017.

15. Souza MC, Mendes IA, Martins JC, de Godoy S, Souza-Junior VD, Trevizan MA, et al. Empathizing and systemizing profiles of Brazilian and Portuguese nursing undergraduates. Nurs Ethics. 2020;27(1):221-9.

16. Vanlaere L, Coucke T, Gastmans C. Experiential learning of empathy in a care-ethics lab. Nurs Ethics. 2010;17(3):325-36.

17. Barraza MA, More C, Calzadilla-Núñez A, Aguilera F, Díaz-Narváez VP. Empatía, componentes de la empatía y declinación empática en estudiantes de nutrición y dietética. Salud (Barranquilla). 2019;35(3):360-73.

18. Ulrich DL, Glendon KJ. Interactive group learning: Strategies for nurse educators. New York: Springer; 2005.

19. Falcone EM, Pinho VD, Ferreira MC, Fernandes CS, D'Augustin JF, Krieger S, et al. Validade convergente do Inventário de Empatia (IE). Psico-USF (Bragança Paulista). 2013;18(2):203-10.

20. Nunnally JC. Psychometric theory. New York: McGraw-Hill; 1978.

21. Petrucci C, La Cerra C, Aloisio F, Montanari P, Lancia L. Empathy in health professional students: A comparative cross-sectional study. Nurse Educ Today. 2016;41:1-5.

22. Penprase B, Oakley B, Ternes R, Driscoll D. Do higher dispositions for empathy predispose males toward careers in nursing? A descriptive correlational design. Nurs Forum. 2015;50(1):1-8.

23. Kusiak GS, Mello LT, Andretta I. Empatia e práticas parentais: a importância dos pais se colocarem no lugar dos filhos. Aletheia. 2019;52(2):8-20.

24. Ward J. The empathy enigma: does it still exist? comparison of empathy using students and standardized actors. Nurse Educ. 2016;41(3):134-8. 